\title{
Mass Preserving Mappings and Image Registration
}

\author{
Steven Haker ${ }^{1}$, Allen Tannenbaum ${ }^{2}$, and Ron Kikinis ${ }^{1}$ \\ 1 Department of Radiology, Surgical Planning Laboratory \\ Brigham and Women's Hospital, Boston, MA 02115 \\ haker@bwh . harvard.edu \\ 2 Departments of Electrical and Computer and Biomedical Engineering \\ Georgia Institute of Technology, Atlanta, GA 30332-0250
}

\begin{abstract}
Image registration is the process of establishing a common geometric reference frame between two or more data sets from the same or different imaging modalities possibly taken at different times. In the context of medical imaging and in particular image guided therapy, the registration problem consists of finding automated methods that align multiple data sets with each other and with the patient. In this paper we propose a method of mass preserving elastic registration based on the Monge-Kantorovich problem of optimal mass transport.
\end{abstract}

\section{Introduction}

In this paper, we propose a method for image warping and elastic registration based on the classical problem of optimal mass transport. The mass transport problem was first formulated by Gaspar Monge in 1781, and concerned finding the optimal way, in the sense of minimal transportation cost, of moving a pile of soil from one site to another. This problem was given a modern formulation in the work of Kantorovich [15, and so is now known as the Monge-Kantorovich problem. This type of problem has appeared in econometrics, fluid dynamics, automatic control, transportation, statistical physics, shape optimization, expert systems, and meteorology 20 .

The registration problem, i.e. the problem of of establishing a common geometric reference frame between two or more data sets, is one of the great challenges in medical imaging. Registration has a substantial recent literature devoted to it, with numerous approaches effective in varying situations. These range from optical flow to computational fluid dynamics [5] to various types of warping methodologies. See [17] for a review of the methods as well as an extensive set of references. See also [21] for a number of recent papers on the subject in the area of brain registration, and [14, 7] and [16 for representative examples of optical flow and elastic deformation model approaches.

The method we introduce in this paper is designed for elastic registration, and is based on an optimization problem built around the $L^{2}$ Monge-Kantorovich distance taken as a similarity measure. The constraint that we put on the transformations considered is that they obey a mass preservation property. We will 
assume that a rigid (non-elastic) registration process has already been applied before applying our scheme. For another application of such mass preserving mappings, see [11.

Our method has a number of distinguishing characteristics. It is parameter free. It utilizes all of the grayscale data in both images, and places the two images on equal footing. It is thus symmetrical, the optimal mapping from image $A$ to image $B$ being the inverse of the optimal mapping from $B$ to $A$. It does not require that landmarks be specified. The minimizer of the distance functional involved is unique; there are no other local minimizers. Finally, it is specifically designed to take into account changes in density that result from changes in area or volume.

We believe that this type of elastic warping methodology is quite natural in the medical context where density can be a key measure of similarity, e.g., when registering the proton density based imagery provided by MR. It also occurs in functional imaging, where one may want to compare the degree of activity in various features deforming over time, and obtain a corresponding elastic registration map. A special case of this problem occurs in any application where volume or area preserving mappings are considered.

\section{Formulation of the Problem}

We now give a modern formulation of the Monge-Kantorovich problem. We assume we are given, a priori, two subdomains $\Omega_{0}$ and $\Omega_{1}$ of $\mathbf{R}^{d}$, with smooth boundaries, and a pair of positive density functions, $\mu_{0}$ and $\mu_{1}$, defined on $\Omega_{0}$ and $\Omega_{1}$ respectively. We assume $\int_{\Omega_{0}} \mu_{0}=\int_{\Omega_{1}} \mu_{1}$ so that the same total mass is associated with $\Omega_{0}$ and $\Omega_{1}$. We consider diffeomorphisms $\tilde{u}$ from $\Omega_{0}$ to $\Omega_{1}$ which map one density to the other in the sense that

$$
\mu_{0}=|D \tilde{u}| \mu_{1} \circ \tilde{u}
$$

which we will call the mass preservation (MP) property, and write $\tilde{u} \in M P$. Equation (1) is called the Jacobian equation. Here $|D \tilde{u}|$ denotes the determinant of the Jacobian map $D \tilde{u}$, and o denotes composition of functions. In particular, Equation (1) implies that if a small region in $\Omega_{0}$ is mapped to a larger region in $\Omega_{1}$, then there must be a corresponding decrease in density in order for the mass to be preserved.

There may be many such mappings, and we want to pick out an optimal one in some sense. Accordingly, we define the squared $L^{2}$ Monge-Kantorovich distance as follows:

$$
d_{2}^{2}\left(\mu_{0}, \mu_{1}\right)=\inf _{\tilde{u} \in M P} \int\|\tilde{u}(x)-x\|^{2} \mu_{0}(x) d x .
$$

An optimal MP map is a map which minimizes this integral while satisfying the constraint (1). The Monge-Kantorovich functional (2) is seen to place a penalty on the distance the map $\tilde{u}$ moves each bit of material, weighted by the 
material's mass. A fundamental theoretical result [410], is that there is a unique optimal $\tilde{u} \in M P$ transporting $\mu_{0}$ to $\mu_{1}$, and that this $\tilde{u}$ is characterized as the gradient of a convex function $w$, i.e., $\tilde{u}=\nabla w$. This theory translates into a practical advantage, since it means that there are no non-global minima to stall our solution process.

\section{Computing the Transport Map}

There have been a number of algorithms considered for computing an optimal transport map. For example, methods have been proposed based on linear programming 20], and on Lagrangian mechanics closely related to ideas from the study of fluid dynamics 3]. An interesting geometric method has been formulated by Cullen and Purser [6].

Let $u: \Omega_{0} \rightarrow \Omega_{1}$ be an initial mapping with the mass preserving (MP) property. Inspired by [49], we consider the family of MP mappings of the form $\tilde{u}=u \circ s^{-1}$ as $s$ varies over MP mappings from $\Omega_{0}$ to itself, and try find an $s$ which yields a $\tilde{u}$ without any curl, that is, such that $\tilde{u}=\nabla w$. Once such an $s$ is found, we will have the Monge-Kantorovich mapping $\tilde{u}$. We will also have $u=\tilde{u} \circ s=(\nabla w) \circ s$, known as the polar factorization of $u$ with respect to $\mu_{0}$ [4].

\subsection{Removing the Curl}

Our method assumes that we have found and initial MP mapping $u$. This can be done for general domains using a method of Moser [18 8], or for simpler domains using a type of histogram specification; see [13. Once an initial MP $u$ is found, we need to apply the process which will remove its curl. It is easy to show that the composition of two mass preserving (MP) mappings is an MP mapping, and the inverse of an MP mapping is an MP mapping. Thus, since $u$ is an MP mapping, we have that $\tilde{u}=u \circ s^{-1}$ is an MP mapping if

$$
\mu_{0}=|D s| \mu_{0} \circ s .
$$

In particular, when $\mu_{0}$ is constant, this equation requires that $s$ be area or volume preserving.

Next, we will assume that $s$ is a function of time, and determine what $s_{t}$ should be to decrease the $L^{2}$ Monge-Kantorovich functional. This will give us an evolution equation for $s$ and in turn an equation for $\tilde{u}_{t}$ as well, the latter being the most important for implementation. By differentiating $\tilde{u} \circ s=u$ with respect to time, we find

$$
\tilde{u}_{t}=-D \tilde{u} s_{t} \circ s^{-1}
$$

while differentiating (3) with respect to time yields $\operatorname{div}\left(\mu_{0} s_{t} \circ s^{-1}\right)=0$, from which we see that $s_{t}$ and $\tilde{u}_{t}$ should have the following forms:

$$
s_{t}=\left(\frac{1}{\mu_{0}} \zeta\right) \circ s, \quad \tilde{u}_{t}=-\frac{1}{\mu_{0}} D \tilde{u} \zeta,
$$


for some vector field $\zeta$ on $\Omega_{0}$, with $\operatorname{div}(\zeta)=0$ and $\langle\zeta, \boldsymbol{n}\rangle=0$ on $\partial \Omega_{0}$. Here $\boldsymbol{n}$ denotes the normal to the boundary of $\Omega_{0}$. This last condition ensures that $s$ remains a mapping from $\Omega_{0}$ to itself, by preventing the flow of $s$, given by $s_{t}=\left(\frac{1}{\mu_{0}} \zeta\right) \circ s$, from crossing the boundary of $\Omega_{0}$. This also means that the range of $\tilde{u}=u \circ s^{-1}$ is always $u\left(\Omega_{0}\right)=\Omega_{1}$.

Consider now the problem of minimizing the Monge-Kantorovich functional:

$$
M=\int\|\tilde{u}(x)-x\|^{2} \mu_{0}(x) d x .
$$

Taking the derivative with respect to time, and using the Helmholtz decomposition $\tilde{u}=\nabla w+\chi$ with $\operatorname{div}(\chi)=0$, we find from (5) that

$$
-\frac{1}{2} M_{t}=\int\langle\tilde{u}, \zeta\rangle=\int\langle\chi, \zeta\rangle .
$$

Thus, in order to decrease $M$, we can take $\zeta=\chi$ with corresponding formulas (5) for $s_{t}$ and $\tilde{u}_{t}$, provided that we have $\operatorname{div}(\chi)=0$ and $\langle\chi, \boldsymbol{n}\rangle=0$ on $\partial \Omega_{0}$. Thus it remains to show that we can decompose $\tilde{u}$ as $\tilde{u}=\nabla w+\chi$ for such a $\chi$.

\subsection{Gradient Descent: $\mathbf{R}^{d}$}

We let $w$ be a solution of the Neumann-type boundary problem

$$
\Delta w=\operatorname{div}(\tilde{u}), \quad\langle\nabla w, \boldsymbol{n}\rangle=\langle\tilde{u}, \boldsymbol{n}\rangle \text { on } \partial \Omega_{0},
$$

and set $\chi=\tilde{u}-\nabla w$. It is then easily seen that $\chi$ satisfies the necessary requirements.

Thus, by (5), we have the following evolution equation for $\tilde{u}$ :

$$
\tilde{u}_{t}=-\frac{1}{\mu_{0}} D \tilde{u}\left(\tilde{u}-\nabla \Delta^{-1} \operatorname{div}(\tilde{u})\right) .
$$

This is a first order non-local scheme for $\tilde{u}_{t}$ if we count $\Delta^{-1}$ as minus 2 derivatives. Note that this flow is consistent with respect to the Monge-Kantorovich theory in the following sense. If $\tilde{u}$ is optimal, then it is given as $\tilde{u}=\nabla w$, in which case $\tilde{u}-\nabla \Delta^{-1} \operatorname{div}(\tilde{u})=\nabla w-\nabla \Delta^{-1} \operatorname{div}(\nabla w)=0$ so that by (9), $\tilde{u}_{t}=0$.

\subsection{Gradient Descent: $\mathbf{R}^{2}$}

The situation is somewhat simpler in the $\mathbf{R}^{2}$ case, due to the fact that a divergence free vector field $\chi$ can in general be written as $\chi=\nabla^{\perp} h$ for some scalar function $h$, where $\perp$ represents rotation by $90 \mathrm{deg}$, so that $\nabla^{\perp} h=\left(-h_{y}, h_{x}\right)$. In this case, we solve Laplace's equation with a Dirichlet boundary condition, and derive the evolution equation

$$
\tilde{u}_{t}=\frac{1}{\mu_{0}} D \tilde{u} \nabla^{\perp} \Delta^{-1} \operatorname{div}\left(\tilde{u}^{\perp}\right) .
$$




\subsection{Defining the Warping Map}

Typically in elastic registration, one wants to see an explicit warping which smoothly deforms one image into the other. This can easily be done using the solution of the Monge-Kantorovich problem. Thus, we assume now that we have applied our gradient descent process as described above and that it has converged to the Monge-Kantorovich optimal mapping $\tilde{u}^{*}$. It is shown in [3] that the flow $X(x, t)$ defined by

$$
X(x, t)=x+t\left(\tilde{u}^{*}(x)-x\right)
$$

is the solution to a closely related minimization problem in fluid mechanics and provides appropriate justification for using (11) to define our continuous warping map $X$ between the densities $\mu_{0}$ and $\mu_{1}$.

\section{Implementation and Examples}

We note that even though our non-local method requires that the Laplacian be inverted during each iteration, the problem has been set up specifically to allow for the use of standard fast numerical solvers which use FFT-type methods and operate on rectangular grids [19. In practice, we found that the standard upwinding scheme was helpful for stability. For a local method in the 2D case, see [13].

We illustrate our methods with a pair of examples. In Figures 1 1and2 we show a brain deformation sequence. Two three dimensional MR data sets, acquired at the Brigham and Women's hospital, were used. The first data set was preoperative, the second was acquired during surgery, after craniotomy and opening of the dura. Both were sub-sampled to $128 \times 128 \times 64$ voxels and pre-processed to remove the skull. The Monge-Kantorvich mapping was found using the evolution equation (9) with intensity values as densities. This process took four hours on a single processor Sun Ultra 10. The warp function (11) together with (1) for the intensities were used to find the continuous deformation through time. The first image shows a planar axial slice, while subsequent images show 2D orthogonal projections of the 3D surfaces which constitute the path of the original slice.

The second example shows an application of our method to surface warping. We have used a colon surface here, but the method can be applied to any surface, such as that of the brain. Figure 3 shows a tubular portion of the colon surface obtained from a CT scan. We cut this tubular surface end to end and flattened it into the plane using a conformal mapping technique 12, as shown in Figure 14 It is well known that a surface of non-zero Gaussian curvature can not be flattened by any means without some distortion. The conformal mapping is an attempt to preserve the appearance of the surface through the preservation of angles. However, in some applications it is desirable to be able to preserve areas instead of angles, so that the sizes of surface structures are accurately represented in the plane. The Monge-Kantorovich approach allows us to find such an areacorrect flattening. Specifically, once we have conformally flattened the surface, we define a density $\mu_{0}$ to be the Jacobian of the inverse of the flattening map, 

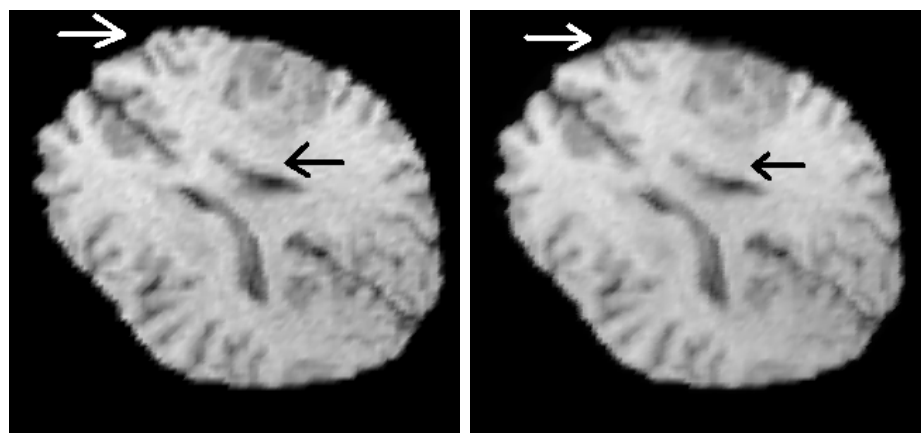

Fig. 1. Brain Warping: $t=0.00$ (left), $t=0.33$ (right). The $t=0.0$ image was given, the $t=0.33$ derived. The arrows indicate the regions of greatest deformation.
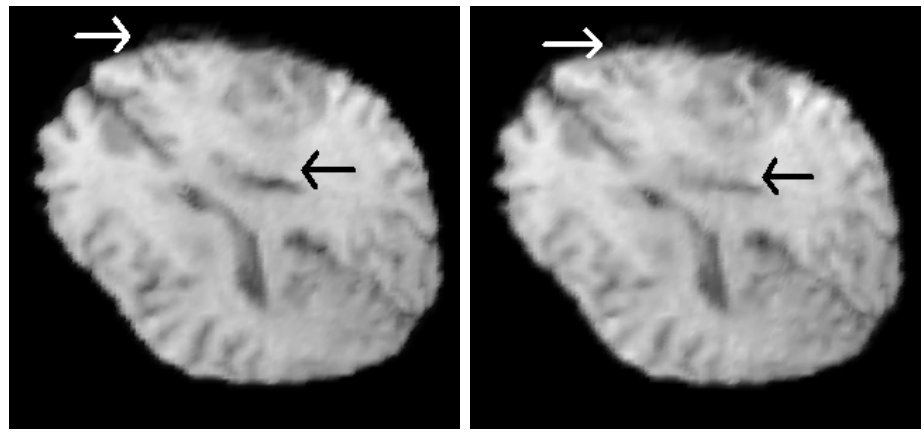

Fig. 2. Brain Warping: $t=0.67$ (left), $t=1.00$ (right). Both images were derived, the final $t=1.00$ image to agree with the target volume according to (11). The arrows indicate the regions of greatest deformation.

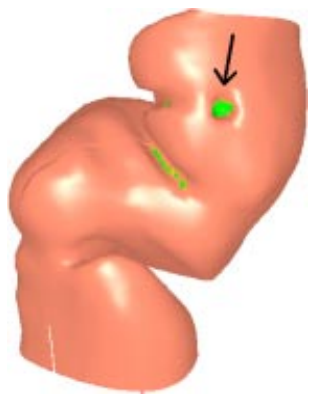

Fig. 3. Original Colon Surface. This portion of colon surface was extracted from CT data. The arrow indicates a phantom polyp. A shading scheme which highlights regions of high Gaussian curvature has been used. 

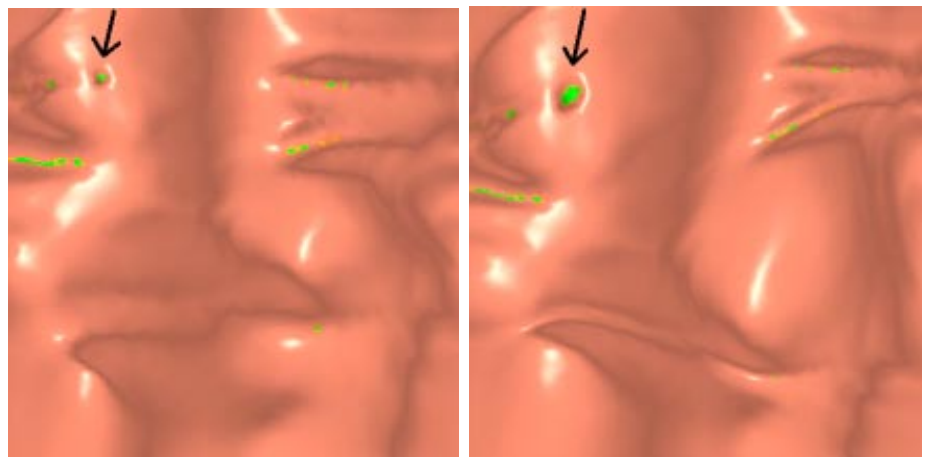

Fig. 4. Conformal (left) and Area Corrected (right) Flattenings. The arrows indicate the phantom polyp. The shading scheme is the same as in Figure 3 , Note that the polyp appears in its true size in the area corrected mapping.

and set $\mu_{1}$ to a constant. The Monge-Kantorovich optimal mapping is then area-correcting by (11). The resulting map took just a few minutes to calculate and is shown in Figure 4. Although corrected for area, surface structures are still clearly discernible. The curl-free nature of the Monge-Kantorovich mapping avoids distortion effects often associated with area preserving maps. There is a circular phantom polyp visible in the upper left corner of the images in Figure4, Note that it is substantially larger in the area-corrected mapping, reflecting its true size.

\section{Conclusions}

In this paper, we presented a natural method for image registration based on the classical problem of optimal mass transportation. Although applied here to the Monge-Kantorovich problem, the method used to enforce the mass preservation constraint is general and has other applications. For example, any weighted linear combination of the Monge-Kantorovich functional and a standard $L^{2}$ energy functional can be used. Gradient descent methods for computing an optimal map in such cases can be derived in a manner very similar to that described above for the pure Monge-Kantorovich functional [1]. Applications of these techniques include brain surface flattening and virtual colonoscopy as described in 212.

Acknowledgments: This work was funded through NIH Grants PO1 CA67165, R01 RR11747, P41 RR13218, and an NIH training grant, as well as grants from AFOSR, ARO, MURI, NSF, Coulter, and NSF-LIS.

\section{References}

1. S. Angenent, S. Haker, A. Tannenbaum, and R. Kikinis, "On area preserving maps of minimal distortion," in System Theory: Modeling, Analysis, and Control, edited by T. Djaferis and I. Schick, Kluwer, Holland, 1999, pp. 275-287. 
2. S. Angenent, S. Haker, A. Tannenbaum, and R. Kikinis, "Laplace-Beltrami operator and brain surface flattening," IEEE Trans. on Medical Imaging 18 (1999), pp. 700-711.

3. J.-D. Benamou and Y. Brenier, "A computational fluid mechanics solution to the Monge-Kantorovich mass transfer problem," Numerische Mathematik 84 (2000), pp. 375-393.

4. Y. Brenier, "Polar factorization and monotone rearrangement of vector-valued functions," Com. Pure Appl. Math. 64 (1991), pp. 375-417.

5. G. E. Christensen, R. D. Rabbit, and M. I. Miller, "Deformable templates using large deformation kinematics," IEEE Trans. of Medical Imag. 5 (1996) pp. 14351447.

6. M. Cullen and R. Purser, "An extended Lagrangian theory of semigeostrophic frontogenesis," J. Atmos. Sci. 41 (1984), pp. 1477-1497.

7. C. Davatzikos, "Spatial transformation and registration of brain images using elastically deformable models," Comp. Vis. and Image Understanding 66 (1997), pp. 207-222.

8. B. Dacorogna and J. Moser, "On a partial differential equation involving the Jacobian determinant," Ann. Inst. H. Poincaré Anal. Non Linéaire, 7 (1990), pp. $1-26$.

9. W. Gangbo, "An elementary proof of the polar factorization of vector-valued functions," Arch. Rational Mechanics Anal. 128 (1994), pp. 381-399.

10. W. Gangbo and R. McCann, "The geometry of optimal transportation," Acta Math. 177 (1996), pp. 113-161.

11. A. F. Goldszal, C. Davatzikos, D. L. Pham, M. X. H. Yan, R. N. Bryan, and S. M. Resnick, "An image processing protocol for qualitative and quantitative volumetric analysis of brain images", J. Comp. Assist. Tomogr., 22 (1998) pp. 827-837.

12. S. Haker, S. Angenent, A. Tannenbaum, and R. Kikinis, "Nondistorting flattening maps and the 3D visualization of colon CT images," IEEE Trans. of Medical Imag., July 2000.

13. S. Haker and A. Tannenbaum, "Optimal transport and image registration," submitted to IEEE Trans. Image Processing, January 2001.

14. N. Hata, A. Nabavi, S. Warfield, W. Wells, R. Kikinis and F. Jolesz, "A volumetric optical flow method for measurement of brain deformation from intraoperative magnetic resonance images," Proc. Second International Conference on Medical Image Computing and Computer-assisted Interventions (1999), pp. 928-935.

15. L. V. Kantorovich, "On a problem of Monge," Uspekhi Mat. Nauk. 3 (1948), pp. 225-226.

16. H. Lester, S. R. Arridge, K. M. Jansons, L. Lemieux, J. V. Hajnal and A. Oatridge, "Non-linear registration with the variable viscosity fluid algorithm," Information Processing in Medical Imaging (1999), pp. 238-251.

17. J. B. A. Maintz and M. A. Viergever, "A survey of medical image registration," Medical Image Analysis 2 (1998), pp. 1-36.

18. J. Moser, "On the volume elements on a manifold," Trans. Amer. Math. Soc. 120 (1965), pp. 286-294.

19. W. Press, S. Teukolsky, W. Vetterling and B. Flannery, Numerical Recipes in C: The Art of Scientific Computing, 2nd Edition, Cambridge University Press, Cambridge U.K., 1992.

20. S. Rachev and L. Rüschendorf, Mass Transportation Problems, Volumes I and II, Probability and Its Applications, Springer, New York, 1998.

21. A. Toga, Brain Warping, Academic Press, San Diego, 1999. 